mouse model of mutant-FLT3-mediated leukaemia, PKC412 treatment completely blocked the development of leukaemia, whereas all of the mice in the placebo group developed fatal disease. Moreover, spleen weights and WBC counts were also significantly lower in the treated mice.

Both of these studies strongly support the idea that FLT3 is potentially a good drug target in AML. PKC412 and CT53518 are now being evaluated in clinical trials for AML, and it seems likely that several other FLT3 inhibitors will also be clinically tested. It will be of considerable interest to compare their efficacies and toxicities, as these are likely to be influenced by the non-FLT3 targets of each drug, which might differ significantly. Peter Kirkpatrick

(2) References and links ORIGINAL RESEARCH PAPERS Kelly, L. M. etal. CT53518, a novel selective FLT3 antagonist for the treatment of acute myelogenous leukemia. Cancer Cell 1, 421-432 (2002) | Weisberg, E. et al. Inhibition of mutant FLT3 receptors in leukemia cells by the small-molecule tyrosine kinase inhibitor PKC412. Cancer Cell 1, 433-443 (2002) FURTHER READING Capdeville, R. et al. Glivec (STI571, imatinib), a rationally developed, targeted anticancer drug. Nature Rev. Drug Discov. 1 493-502 (2002)

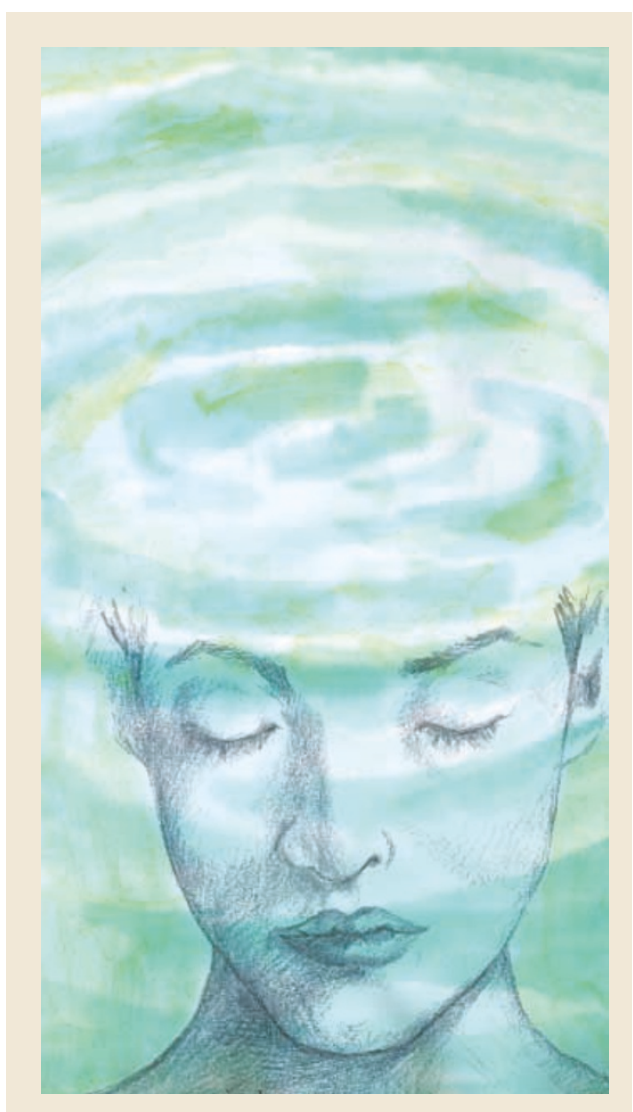

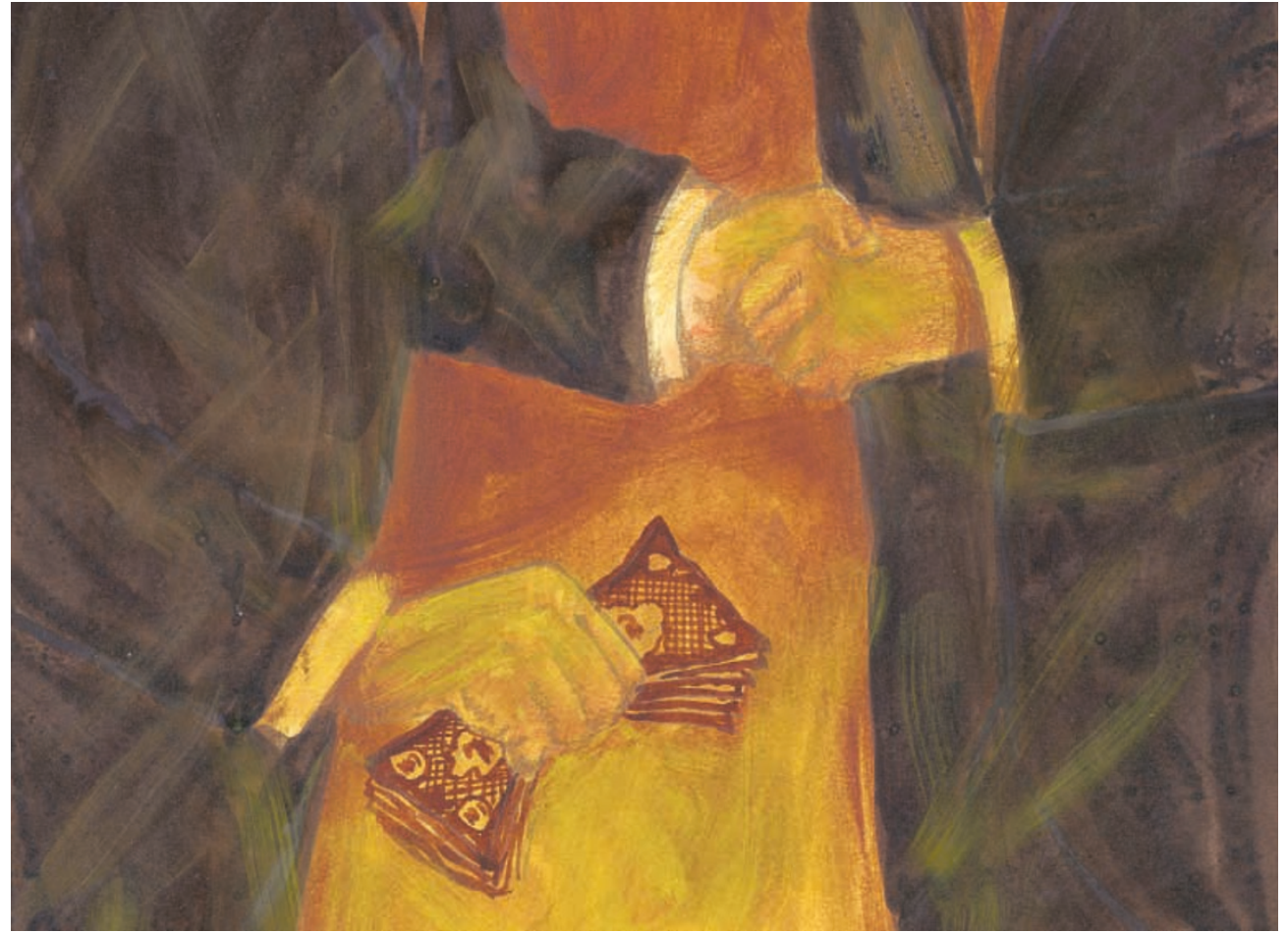

VIRAL INFECTIONS

\title{
Exchange control
}

Exchange is a fundamental process of life. In one type of cellular exchange process, the enzyme thioredoxin and the tripeptide glutathione mediate reduction-oxidation reactions between molecules. Disulphide exchange in one of the molecular domains of the $\mathrm{CD} 4$ molecule is required for entry of HIV-1 into T cells, according to new research in the August issue of Nature Immunology. This redox change could represent new potential targets for HIV-entry inhibitors.

HIV infection of helper $\mathrm{T}$ cells requires the receptor $\mathrm{CD} 4$, as well as a chemokine co-receptor. Although co-receptor use varies between virus strains, the use of the $\mathrm{CD} 4$ receptor is an almost universal feature of $\mathrm{HIV}-1$ strains. CD4 is a surface membrane protein that is expressed at high levels on helper $\mathrm{T}$ cells. The molecule contains four immunoglobulin-like domains D1 to D4 - of which D1, D2 and D4 each contains an intramolecular disulphide bond. These are formed when the sulphydryl group of one cysteine residue reacts with that of another cysteine in an oxidation reaction to form a disulphide bridge. An unusual feature of the D2 disulphide bond is its relatively high strain energy compared with the other disulphide bonds, which makes it more prone to reduction to the free sulphydryl groups.

Hogg and colleagues showed that a fraction of the cell-surface $\mathrm{CD} 4$ molecules contained one or more free thiols, and this was due to redox activity of the D2 disulphide bond. Regulation of the D2 redox state seems to be controlled by thioredoxin, which is secreted by $\mathrm{T}$ cells. The authors tested whether the redox state of the CD4 D2 domain was important for HIV-1 entry. To prevent the exchange between the oxidized and reduced forms, the authors used glutathionearsenoxide, which consists of a trivalent arsenical attached to the cysteine thiol of glutathione. Trivalent arsenicals form high-affinity ring structures with closely spaced dithiols, but not with monothiols or dithiols that are spaced far apart. Fixing the D2 thiols in the reduced state by labelling with this agent blocked HIV entry. It seems, therefore, that conformational changes in the D2 domain after reduction/oxidation and/or disulphidedependent dimerization of $\mathrm{CD} 4$ molecules are important for HIV-1 infection of T cells.

Binding of the envelope protein of HIV-1 to CD4, which occurs through the D1 domain, is not affected by the redox state of the $\mathrm{D} 2$ disulphide. HIV-1 entry, however, occurs preferentially by means of receptors that are in the oxidized state. Detailed quantification of binding kinetics and avidity of HIV for the different redox forms of CD4 might reveal the explanation for the authors' observations. As well as having potential therapeutic applications for HIV-1 infections, this strategy could be a general approach for other molecules that contain redox-active structures. References and links Re Brazt
ORIGINAL RESEARCH PAPER Matthias, L. J. et al. Disulphide
exchange in domain 2 of CD4 is required for entry of HIV-1. Nature Immunol. 2002 Jul 1 (doi: 10.1038/ni819)

FURTHER READING Lay, A. J. et al.Phosphoglycerate kinase acts in tumour angiogensis as a disulphide reductase. Nature 408, 869-873 (2000) | Xie, L. et al. Control of von Willebrand factor multimer size by thrombospondin-1. J. Exp. Med. 193, 1341-1349 (2001)

\section{WEB SITES}

Hogg's laboratory:

http://notes.med.unsw.edu.au/resinterests.nsf/sw/9100604 